OPEN ACCESS

Edited by:

Marie-Agnes Dragon-Durey,

Université Paris Descartes,

France

Reviewed by:

Aleksandra Antovic,

Karolinska Institutet, Sweden

Massimo Dell'Antonia,

University of Cagliari, Italy

*Correspondence:

yi2006liu@163.com

tThese authors have contributed equally to this work

Specialty section: This article was submitted to Autoimmune and Autoinflammatory Disorders, a section of the journal

Frontiers in Immunology

Received: 29 July 2021 Accepted: 13 December 2021 Published: 07 January 2022

Citation:

Tang Z, Wang X, Xia Z, Wang Z, Zhao Y and Liu Y (2022) Case Report:

Multicentric Reticulohistiocytosis Associated With Posterior Mediastinal

Adenosquamous Carcinoma,

Antinuclear Antibody Positivity and Lupus Anticoagulant Positivity.

Front. Immunol. 12:749669. doi: 10.3389/fimmu.2021.749669

\section{Case Report: Multicentric Reticulohistiocytosis Associated With Posterior Mediastinal Adenosquamous Carcinoma, Antinuclear Antibody Positivity and Lupus Anticoagulant Positivity}

\author{
Ziyi Tang ${ }^{1,2+}$, Xiangpeng Wang ${ }^{1 \dagger}$, Zijing Xia ${ }^{1,2+}$, Zhongming Wang ${ }^{1}$, \\ Yi Zhao ${ }^{1}$ and Yi Liu ${ }^{1,2 *}$
}

\begin{abstract}
1 Department of Rheumatology and Immunology, West China Hospital, Sichuan University, Chengdu, China, ${ }^{2}$ Laboratory of Rheumatology and Immunology, West China Hospital, Sichuan University, Chengdu, China
\end{abstract}

Multicentric reticulohistiocytosis $(\mathrm{MRH})$ is a rare systemic disease of non-Langerhans cell histiocytosis. A number of studies in the literature have documented that it can coexist with malignancy or autoimmune disease, making it difficult to determine the most appropriate therapy. Here, we present a case study of $\mathrm{MRH}$ associated with posterior mediastinal adenosquamous carcinoma along with antinuclear antibody positivity and lupus anticoagulant positivity. The patient experienced 6 months of clinical benefit after surgical resection and chemoradiotherapy of the mediastinal malignancy. This case adds to the available literature on multicentric reticulohistiocytosis associated with different types of malignancy and provides supplementary clinical data on the coexistence of this syndrome with malignancy and immune system abnormalities. To the best of our knowledge, this is the first case study describing $\mathrm{MRH}$ accompanied by posterior mediastinal adenosquamous carcinoma and lupus anticoagulant positivity. The unknown aetiology and polymorphic clinical presentation of $\mathrm{MRH}$ warrants further investigation.

Keywords: multicentric reticulohistiocytosis, mediastinal malignancy, paraneoplastic syndrome, rare disease, case report

\section{INTRODUCTION}

Multicentric reticulohistiocytosis (MRH), also known as lipoid dermatoarthritis, is a rare systemic disease of non-Langerhans cell histiocytosis (N-LCH). The disease was first systematically reported in 1937 by Weber \& Freudenthal, and there have been no more than 400 cases reported globally, mostly presenting in middle-aged white women $(1,2)$. The main manifestations of MRH are destructive polyarthritis and papulonodular skin lesions that occasionally affect other organs, including the lungs, heart, liver and kidneys (3). The aggressive erosion of the distal interphalangeal joints generally 
precedes the presentation of the skin phenotype and is the most frequent manifestation of $\mathrm{MRH}$; thus, it can be used as the basis for differential diagnosis. Skin lesions most frequently appear on the hands and face, including flesh-coloured to pinkish papules and nodules. The appearance of "coral beads" around the nail folds is a characteristic sign of MRH. Dermatoscopy of these lesions were reported to show some typical features: a pattern with various shades of yellow/orange/reddish (like the "setting-sun" pattern), central white scar-like patches and streaks, brown reticular structures and linear teleangectasias, which may be a clue to diagnosis (3-6). MRH patient skin biopsies typically show a large degree of lymphohistiocytic proliferation and multinucleated giant cells with fine granules and granular ground glass-like cytoplasm. These features are used to establish a diagnosis of MRH by histology. Unfortunately, the molecular pathogenesis of MRH remains poorly understood. Alternatively, activated macrophages, elevated proinflammatory cytokines and osteoclast activation may be involved in the affected tissues $(7,8)$.

Some researchers hold the view that $\mathrm{MRH}$ is a neoplastic or paraneoplastic disease, and approximately $25 \%$ of $\mathrm{MRH}$ patients do indeed have an underlying malignancy (9). On the other hand, an analysis of the literature reveals that approximately $15 \%-29 \%$ of MRH cases coexist with autoimmune diseases such as Sjogren's syndrome, rheumatoid arthritis, systemic lupus erythematosus, systemic sclerosis, dermatomyositis, and undifferentiated connective tissue disease $(9,10)$. Several cases of MRH-concomitant thrombosis have also been reported (9, 11-13). However, the relationship among $\mathrm{MRH}$, malignancy, autoimmune disease, and thrombosis remains unclear because of the rarity of the disease. It therefore remains to be determined whether these are mere associations or truly part of the disease course. There is no appropriate treatment for $\mathrm{MRH}$, and several therapeutic agents have been used with varying efficacy. Here, we describe the case of a patient who had posterior mediastinal adenosquamous carcinoma with positive antinuclear antibody (ANA) and lupus anticoagulant (LA) positivity; the patient experienced 6 months of clinical benefit after surgical resection and chemoradiotherapy for the mediastinal malignancy.

\section{CASE DESCRIPTION}

A previously healthy, 36-year-old Chinese man presented with papulonodules on his face and hands that had emerged over an 8-month period, along with experiencing polyarthritis for half a year. He reported smoking half a pack per day for 17 years. His family history was significant for malignancy in that his mother had breast cancer.

On admission, a physical examination revealed multiple fleshcoloured to pinkish papules and nodules on his face (Figure 1A), distal interphalangeal and proximal interphalangeal areas, and papulonodular lesions arranged around the nail plica in a typical "coral bead" pattern (Figures 1B, C). The cutaneous lesions were tender but without pruritus. There was also significant tenderness noted in the bilateral interphalangeal joints, wrists, elbows, shoulders, ankles, knees and hips, with no signs of swelling.
Laboratory tests showed slightly elevated inflammatory markers, including a C-reactive protein (CRP) level of 23.4 $\mathrm{mg} / \mathrm{L}$ (reference range, $<5 \mathrm{mg} / \mathrm{L}$ ), erythrocyte sedimentation rate (ESR) of $49 \mathrm{~mm} / \mathrm{h}$ (reference range, $<21 \mathrm{~mm} / \mathrm{h}$ ), interleukin-6 (IL6) level of $9.69 \mathrm{pg} / \mathrm{ml}$ (reference range, $0-7 \mathrm{pg} / \mathrm{ml}$ ) and tumour necrosis factor (TNF) level of $9.26 \mathrm{pg} / \mathrm{ml}$ (reference range, $<7 \mathrm{pg}$ / $\mathrm{ml}$ ). Routine blood, biochemistry, urinalysis, C3 and C4 levels were normal. The levels of tumour markers (CEA, AFP, CA125, CA19-9, CA72-4, CA15-3, CYFRA21-1 and PSA) did not exceed the reference range. Further analysis revealed that the patient showed ANA (titer 1:320), LA (1.93, reference range, 0.8-1.2, LA performed with dilute Russell's viper venom time [dRVVT] screen/confirm), PPD test and tuberculosis interferon- $\gamma$ release assay positivity. The level of fibrinogen was $5.47 \mathrm{~g} / \mathrm{L}$ (reference range, 2.0-4.0 g/L). Tests for anti-dsDNA, anti-Smith, anticentromere, anti-ribonucleoprotein, anti-Jo1, anti-neutrophil cytoplasmic antibodies, rheumatoid factor (RF), anti-cyclic citrullinated peptide antibodies (ACPA), anticardiolipin antibodies (IgG, IgM and IgA), anti- $\beta 2$-glycoprotein I antibodies (IgG, IgM and IgA) and HbsAg were all negative. Radiographs of the joints revealed osteoporosis in both hands and wrists and a slightly narrowed space between the first and second metacarpophalangeal joints of both hands (Supplementary Material 1). High-resolution computed tomography (HRCT) of the chest revealed emphysema and an inconspicuous mass of lymphoglandula within the posterior mediastinum (Figure 1D). The patient underwent endobronchial ultrasound (EBUS) puncture biopsy of the mediastinal lymphoglandula to exclude cancer after consultation with the Department of Respiratory Medicine. Contrast-enhanced CT imaging of the whole abdomen revealed calcified plaques in the abdominal aorta and its branches, with mural thrombosis. The above indicators led to a presumptive diagnosis of undifferentiated connective tissue disease and possible antiphospholipid syndrome. On day 7, the histopathologic features of the papulonodular skin lesions showed proliferation of abundant foamy macrophages and the presence of histocytes (Figure 1E). These lesions contained CD68-positive cells (Figure 1F) that were negative for S100, CD1a, Langerin, and Factor VIII markers (Supplementary Material 2). Thus, a histological diagnosis of $\mathrm{MRH}$ was made.

The patient received methotrexate (15 $\mathrm{mg}$ once a week) accompanied by oral prednisone (10 mg twice a day) and was discharged after 20 days of treatment. He continued the above regimen after discharge according to his doctor's instructions. Approximately one month later, he was admitted again for assessment, which indicated that the papulonodular lesions had shrunk and that the inflammatory cytokines and molecules described above had returned to normal levels. Nonetheless, the patient's joint pain was not significantly relieved.

The results of EBUS puncture biopsy of the mediastinal lymphoglandula were available at the second hospital admission and showed a small number of abnormal cells (Supplementary Material 3). Immunohistochemistry showed cells positive for PCK, EMA, CK5/6 (focal), GATA-3, Ki67 and MIB-1 (20\% positive). CR, TTF-1, P63, D2-40 and CgA were 


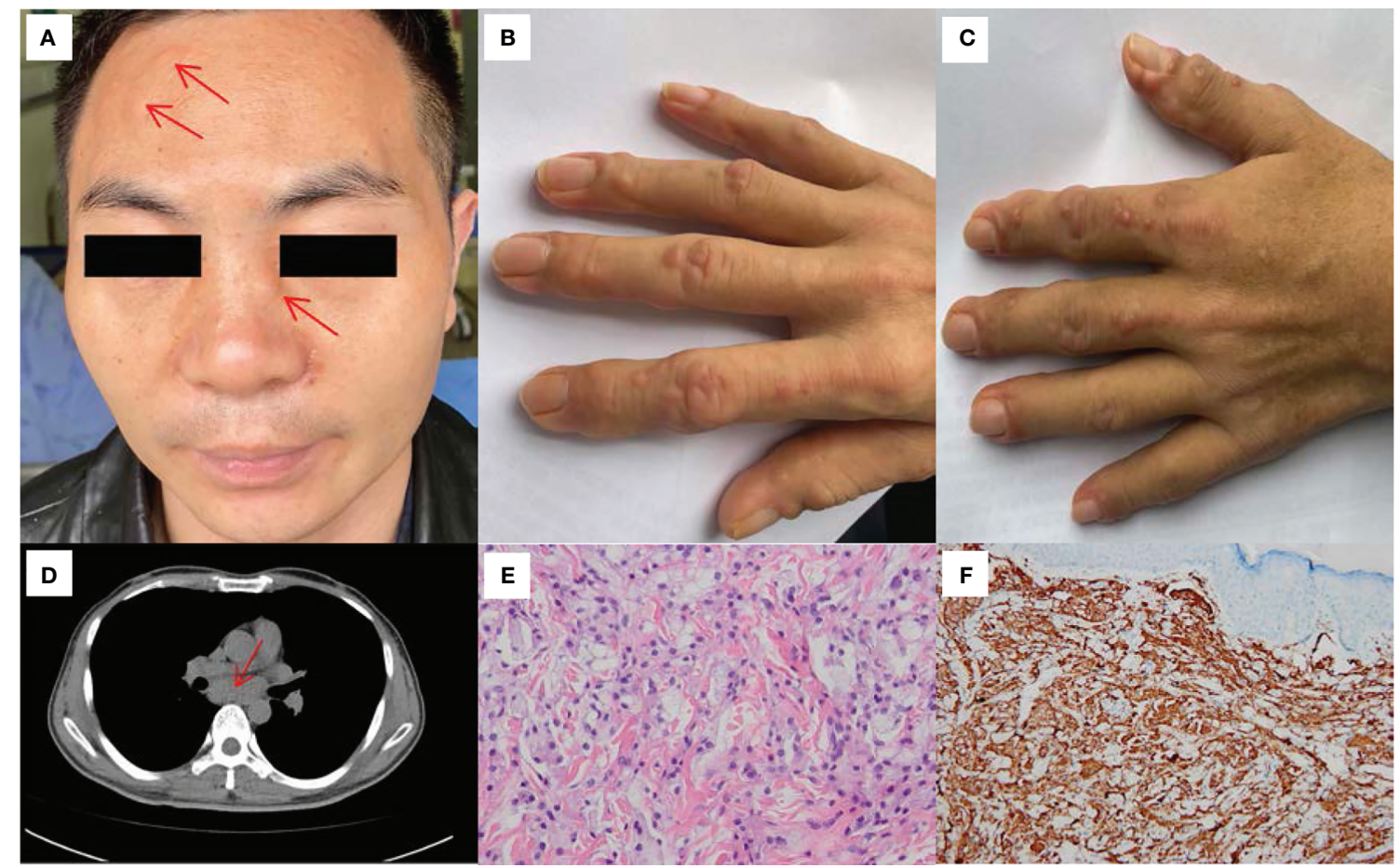

FIGURE 1 | Clinical, HRCT and histological features of our patient. (A) Multiple flesh-colored to pinkish papules and nodules were noted on his face (before treatment). (B) Flesh-colored to pinkish papulonodular lesions were observed on the right hand (before treatment). (C) Flesh-colored to pinkish papulonodular lesions were observed on the left hand (before treatment). (D) HRCT of the chest revealed a mass of lymphoglandula within the posterior mediastinum. (E) Biopsy of papule skin lesions showed proliferation of abundant foamy macrophages and histocytes in the dermis (HEx400). (F) Dermal and epidermotropic foamy macrophages were strongly positive for CD68 $(\times 200)$.

negative, and a diagnosis of epithelial neoplasia was made. To identify the primary lesion, fluorodeoxyglucose positron emission tomography/CT (FDG-PET/CT) was carried out, revealing increased glycometabolism in the soft tissue around the joints of the extremities and the mediastinal lymph nodes; the subcarinal lymph node findings confirmed the suspicion of malignancy (Figure 2). However, the primary tumour was not found despite exhaustive tests. The patient discontinued methotrexate and prednisone according to the recommendations of the multidisciplinary treatment (MDT) team.

To further clarify the diagnosis and treatment approach, the patient received video-assisted thoracoscopic surgery (VATS) to excise the mediastinal mass $(6 \times 3.5 \times 2.8 \mathrm{~cm})$ for genetic analysis. Malignant cells were identified in the background of abundant lymphoid tissue in the mediastinal mass (Supplementary Material 4). Histopathologic features of malignant cells were positive for P63 (focal), CK5/6 (focal), PCK, GATA-3, and Ki-67 (MIB-1, 50-60\%) but not CR, PAX-8, CD5, CD117, WT-1, CgA, or EBER1/2, resulting in a diagnosis of poorly differentiated adenosquamous carcinoma. Genetic analyses revealed the presence of mutations in the SMARCA gene A4 (NM_003072.3:c.2438+1G>A IVS16, mutation rate 36.4\%), amplification of EGFR, and a lack of PD-L1.
After discussion with oncologists, chemoradiotherapy was administered, namely, intravenous paclitaxel $210 \mathrm{mg}$ for 1 day combined with intravenous carboplatin $450 \mathrm{mg}$ for 1 day (once every 3 weeks) and radiotherapy for the mediastinal lymph nodes. After 6 months of follow-up from the diagnosis of malignancy (5 cycles of chemotherapy and 30 cycles of radiotherapy), the patient's joint pain was significantly reduced, and the papulonodular lesions had almost cleared, without any specific treatment. The latest re-examination on June 29, 2021, revealed that the patient was ANA negative and the LA level had decreased to 1.32, with no signs of tumour recurrence. The patient is still being followed-up. The changes to the lesions on the hands are presented in Supplementary Material 5. The patient's clinical course is shown in Figure 3.

\section{DISCUSSION}

$\mathrm{N}-\mathrm{LCH}$ consists of a group of rare diseases with different clinical presentations, pathogeneses and morphologies, including $\mathrm{MRH}$, Erdheim-Chester disease, and Rosai-Dorfman disease, among others (14). MRH can be differentiated from Erdheim-Chester disease and Rosai-Dorfman disease by its pathological and clinical manifestations. In our patient, a diagnosis of MRH was 


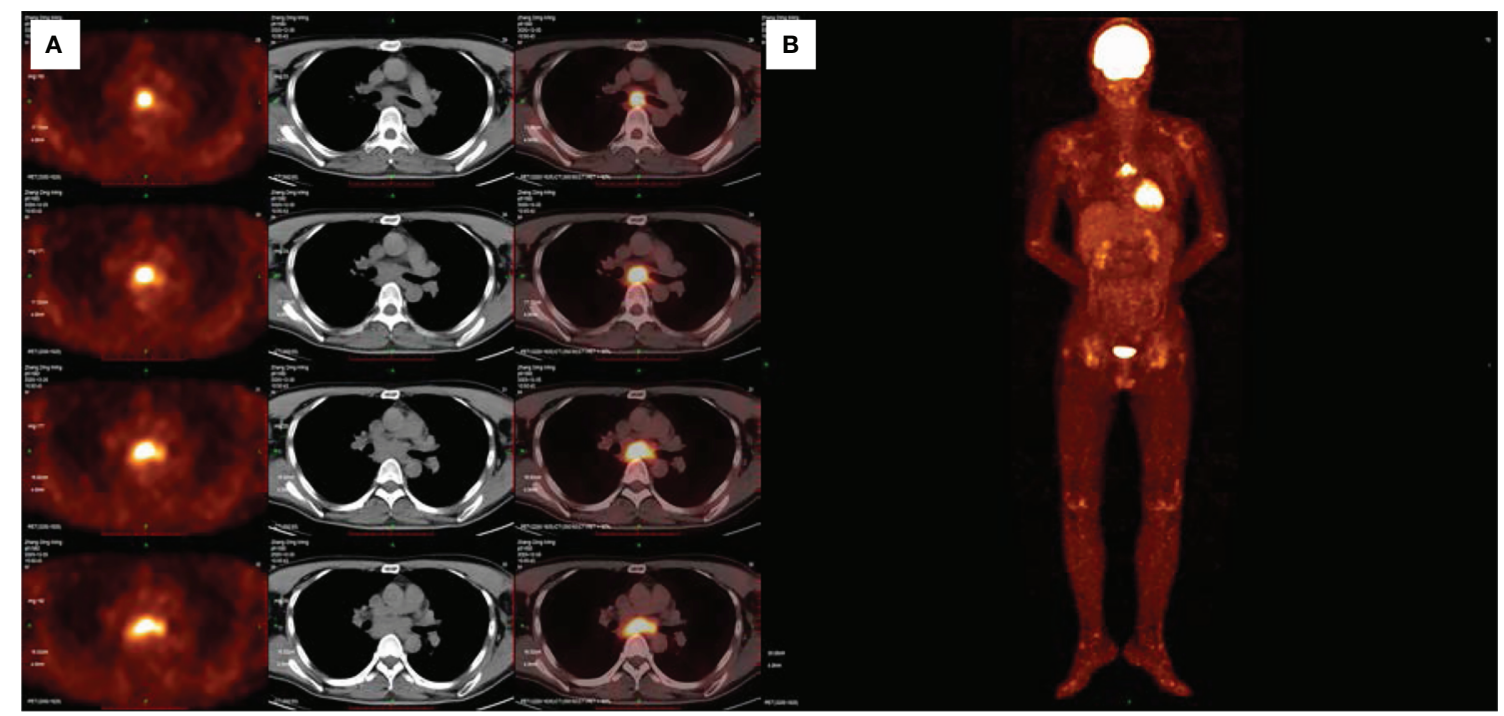

FIGURE 2 | FDG-PET/CT showing increased glycometabolism in the mediastinal lymph nodes and soft tissue around the joints of the extremities (A, B).

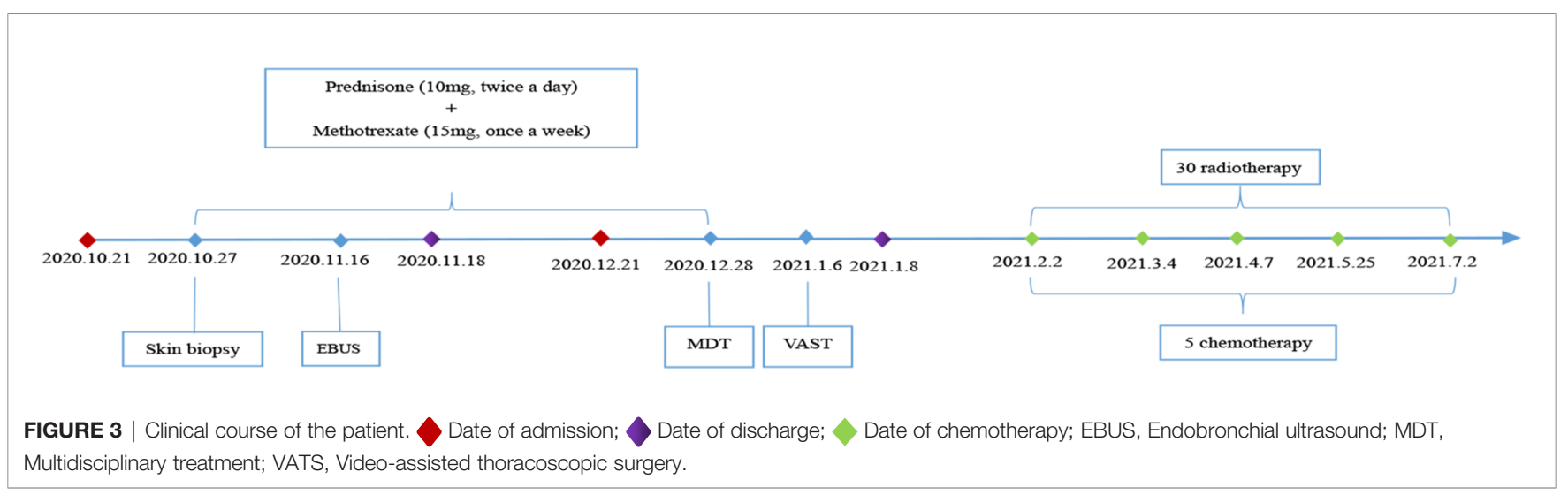

made based on the typical skin presentation and skin lesion histology, but destructive arthritis was not observed at that time. This could be attributed to the very early initiation of treatment with disease-modifying drugs. Although inflammatory markers have been suggested to play a role in the development of disease, they seem to have had little special value for diagnosis; increased levels of cytokines have been reported in the literature (15), but the majority of cases have levels within the normal range.

The neoplastic and autoimmune nature of the disease has been a focus of previous MRH case reports since the first was published in 1937. MRH-related malignancies can involve various systems and organs, including the breast, bloodstream, gastrointestinal organs, gynaecological organs, lung, and skin. A previous study described an MRH patient presenting with two primary cancers, and metastatic occult cancer in the left axilla has also been reported $(16,17)$. Additionally, two cases of mediastinal tumours have been reported, both of which were thymomas of the anterior chest $(18,19)$. The present report of posterior mediastinal malignancy appears to be the first. Clinically, apart from primary thymic/thyroid masses and lymphomas, all other mediastinal masses can be considered rare tumours, and posterior mediastinal masses are generally neurogenic tumours, followed by sarcomas (20). This means that our case is extremely rare.

Another concern in our case is mutated SMARCA4. In the analysed literature, SMARCA4 encodes one of the enzymatic (ATPase) subunits of the mammalian SWI/SNF family, a chromatin remodelling complex that directs nucleosomes and modulates gene expression. The gene is located on chromosome 19p13.2 and encodes the BRG1 protein, inactivating mutations of which result in its loss of expression and characterize a group of malignancies (21). Thus, we introduce a rare malignancy, called SMARCA4-deficient thoracic tumour (SMARCA4-DTT), a distinct entity of undifferentiated thoracic malignancy that was 
first described in 2015 (22), presenting aggressive behaviour with compressive or infiltrative features and conferring a poor prognosis. SMARCA4-DTT mainly involves the mediastinum, lung, and/or pleura and mostly occurs in heavy smokers with emphysema; it requires complete identification and differentiation from other epithelial malignancies involving the thoracic region, and fortunately, many physicians are becoming increasingly aware of this disease $(23,24)$. The majority of SMARCA4-DTTs have been reported as SMARCA4-deficient thoracic sarcomas thus far, but an increasing number of carcinomas have been reported (25). We were surprised to find that the malignancy presentation in our patient was highly similar to SMARCA4-DTT. There is currently no recognized diagnostic standard for SMARCA4-DTT, and our case may provide a reference for future research.

At present, whether $\mathrm{MRH}$ is a paraneoplastic syndrome remains controversial. We do find that some patients, such as the one described in this case, experience remission of the lesions after anti-tumour treatment, or the onset of MRH occurs just prior to relapse. Nevertheless, the fact that malignancies are observed in association with $\mathrm{MRH}$ and the fact that these two diseases do not always run a parallel course makes the paraneoplastic nature of this entity questionable. A Japanese study performed whole-exome sequencing and RNA sequencing in two patients with $\mathrm{MRH}$, and the results indicated that MRH should be considered a neoplastic disease caused by activation of the RAS-MAPK pathway and that the effects of chemotherapy on this disease are promising (26).

Our patient experienced a very insidious onset of malignancy. Due to the background of negative tumour markers in routine serum tests and the lack of symptoms, it can be difficult to detect this type of malignancy by chest HRCT, especially by inexperienced clinicians. In recent years, the application of FDG-PET/CT in MRH has attracted increasing attention (2729). FDG-PET/CT is a potentially useful way to detect and evaluate the grade of inflammatory involvement of $\mathrm{MRH}$ and to assess the possible associations with malignant neoplasms, as FDG is easily taken up by granulomas and inflammatory cells.

In case reports, MRH combined with immune diseases is common, and ANA, ACPA and RF positivity can be observed, but laboratory testing showing LA positivity is unusual. This is the first report of MRH in which ANA and LA were both found to be positive. Since our patient only had mural thrombosis in the abdominal aorta and its branches, no anticoagulants were administered. Due to the lack of clinical manifestations and other specific serological indicators, connective tissue disease could not be diagnosed in our case. After surgical resection and chemoradiotherapy for the malignancy, we observed that the ANA of the patient became negative and there was a significant decrease in LA. This reminded us to consider a positive result of ANA and LA in our case dialectically because studies have shown that ANA and LA are associated with malignancy (30-33).

Given that its clinical manifestations are similar to rheumatic conditions, it has been proposed that $\mathrm{MRH}$ is an autoimmune or inflammatory disease, so treatments such as the administration of corticosteroids, methotrexate, thalidomide (34), bisphosphonates, and biological anti-inflammatory agents [etanercept, adalimumab (35), infliximab, tofacitinib (36), upadacitinib (37)] have been employed with varying efficacy. Although our patient was sensitive to methotrexate treatment, we had to discontinue it because of the malignancy. The main focus of treatment was to prevent joint damage, but priority had to be given to the management of the malignancy once it had been diagnosed. It is worth noting that $\mathrm{MRH}$ can be associated with either immune disease or malignancy, making the selection of an appropriate therapeutic regimen challenging because the two possibilities are mutually exclusive. In our patient's case, MRH remission was observed after treatment of the malignancy without any other specific treatment, although his long-term prognosis is still unknown.

In conclusion, although a definitive diagnosis of $\mathrm{MRH}$ rests upon histological examination of biopsy specimens, careful roentgenological interpretation and analysis of the clinical features are the keys to early diagnosis of this disease. The types of malignancies that can be complicated by $\mathrm{MRH}$ are diverse and lack specificity, and once diagnosed, patients with $\mathrm{MRH}$ warrant thorough examination. FDG-PET/CT should be recommended as an essential procedure to exclude underlying malignancy, regardless of whether tumour markers show negativity. At the same time, screening for immune system diseases and evaluation of the risk of thrombosis should not be neglected. Due to the high incidence of joint destruction, systemic involvement and malignancy, MRH needs to be recognized and given more clinical attention. The current study of this disease is insufficient; we believe that this case report will provide guidance to medical practitioners encountering similar clinical presentations.

\section{DATA AVAILABILITY STATEMENT}

The original contributions presented in the study are included in the article/Supplementary Material. Further inquiries can be directed to the corresponding author.

\section{ETHICS STATEMENT}

The studies involving human participants were reviewed and approved by Sichuan University West China Hospital Health Research Ethics. Written informed consent for participation was not required for this study in accordance with the national legislation and the institutional requirements. Written informed consent was obtained from the individual(s) for the publication of any potentially identifiable images or data included in this article.

\section{AUTHOR CONTRIBUTIONS}

ZT, XW, and ZX collected clinical data, summarized the case, reviewed the literature, and drafted the manuscript. $\mathrm{ZW}$ and $\mathrm{YZ}$ 
provided the case. YL reviewed and summarized the case. All authors contributed to the article and approved the submitted version.

\section{FUNDING}

This work was supported by 1.3 .5 project for disciplines of excellence, West China Hospital, Sichuan University (Grant Number: ZYGD18015, ZYJC18003).

\section{REFERENCES}

1. Kumar B, Singh N, Rahnama-Moghadam S, Wanat KA, Ijdo JW, Werth VP. Multicentric Reticulohistiocytosis: A Multicenter Case Series and Review of Literature. J Clin Rheumatol (2018) 24:45-9. doi: 10.1097/ rhu.0000000000000594

2. Barrow MV, Holubar K. Multicentric Reticulohistiocytosis. A Review of 33 Patients. Med (Baltimore) (1969) 48:287-305. doi: 10.1097/00005792196907000-00002

3. Orlando G, Dan G, Pezzetta S, Linder D, Salmaso R. Coral-Bead Skin Lesions Associated With Erosive Arthritis: A Quiz. Acta Derm Venereol (2019) 99:844-5. doi: 10.2340/00015555-3210

4. Kaçar N, Tasli L, Argenziano G, Demirkan N. Reticulohistiocytosis: Different Dermatoscopic Faces and a Good Response to Methotrexate Treatment. Clin Exp Dermatol (2010) 35(4):e120-2. doi: 10.1111/j.1365-2230.2009.03738.x

5. Sobjanek M, Sławińska M, Romaszkiewicz A, Sokołowska-Wojdyło M, JasielWalikowska E, Nowicki R. Dermoscopic Features of Periungual Papules in Multicentric Reticulohistiocytosis. J Eur Acad Dermatol Venereol (2017) 31 (10):e442-e3. doi: 10.1111/jdv.14260

6. Dell'Antonia M, Atzori L, Pilloni L, Ferreli C. Multicentric Reticulohistiocytosis Revealing Breast Cancer: Report of a Case With Dermoscopic, Radiological and Therapeutic Aspects. Australas J Dermatol (2021) 62(4):527-9. doi: 10.1111/ajd.13687

7. Kamiya K, Komine M, Murata S, Ohtsuki M. Involvement of M2 Macrophages in the Pathomechanisms of Multicentric Reticulohistiocytosis. Int J Dermatol (2017) 56:e173-5. doi: 10.1111/ijd.13661

8. Selmi C, Greenspan A, Huntley A, Gershwin ME. Multicentric Reticulohistiocytosis: A Critical Review. Curr Rheumatol Rep (2015) 17:511. doi: 10.1007/s11926-015-0511-6

9. Toz B, Büyükbabani N, İnanç M. Multicentric Reticulohistiocytosis: Rheumatology Perspective. Best Pract Res Clin Rheumatol (2016) 30:25060. doi: 10.1016/j.berh.2016.07.002

10. Sanchez-Alvarez C, Sandhu AS, Crowson CS, Wetter DA, McKenzie GA, Lehman JS, et al. Multicentric Reticulohistiocytosis: The Mayo Clinic Experience (1980-2017). Rheumatol (Oxford) (2020) 59:1898-905. doi: 10.1093/rheumatology/kez555

11. Olabi B, Abbas A, Shah J, Tidman MJ, Ayob S, Abhishek A. Multicentric Reticulohistiocytosis: An Association With Chronic Sagittal Sinus Thrombosis. Clin Exp Dermatol (2021) 46:554-6. doi: 10.1111/ced.14433

12. Rentsch JL, Martin EM, Harrison LC, Wicks IP. Prolonged Response of Multicentric Reticulohistiocytosis to Low Dose Methotrexate. J Rheumatol (1998) 25:1012-5

13. Malhotra K, Mazumder R, Liebeskind DS. Multicentric Reticulohistiocytosis: An Unknown Source of Embolic Stroke. J Stroke Cerebrovasc Dis (2017) 26: e22-4. doi: 10.1016/j.jstrokecerebrovasdis.2016.10.023

14. Mavrogenis AF, Igoumenou VG, Antoniadou T, Megaloikonomos PD, Agrogiannis G, Foukas P, et al. Rare Diseases of Bone: Erdheim-Chester and Rosai-Dorfman non-Langerhans Cell Histiocytoses. EFORT Open Rev (2018) 3:381-90. doi: 10.1302/2058-5241.3.170047

15. Bennàssar $\mathrm{A}$, Mas $\mathrm{A}$, Guilabert $\mathrm{A}$, Julià $\mathrm{M}$, Mascaró-Galy JM, Herrero $\mathrm{C}$. Multicentric Reticulohistiocytosis With Elevated Cytokine Serum Levels. J Dermatol (2011) 38:905-10. doi: 10.1111/j.1346-8138.2010.01146.x

16. Ge JS, Hung SJ, Hsu YH, Hung JS. Rare Case of Multicentric Reticulohistiocytosis Presenting as Epidermotropic Papular Xanthoma and

\section{ACKNOWLEDGMENTS}

The authors wish to thank the patient in this study.

\section{SUPPLEMENTARY MATERIAL}

The Supplementary Material for this article can be found online at: https://www.frontiersin.org/articles/10.3389/fimmu.2021. 749669/full\#supplementary-material

Double Primary Cancers. J Dermatol (2018) 45:e138-9. doi: 10.1111/13468138.14182

17. Worm M, Kleine-Tebbe A, Von Stebut E, Haas N, Kolde G. Multicentric Reticulohistiocytosis Indicating Metastasis of an Unknown Primary Tumour. Acta Derm Venereol (1998) 78:67-8. doi: 10.1080/00015559850135896

18. Shwe S, Sharma A, Elsensohn AN, Doan L, Smith J. Multicentric Reticulohistiocytosis in a Patient With Thymic Carcinoma. JAAD Case Rep (2019) 5:720-2. doi: 10.1016/j.jdcr.2019.06.012

19. Rudha Y, Starobinska E, Abdulqader Y, Attya A, Guerrero C, Parperis K. Multicentric Reticulohistiocytosis Associated With Thymic Carcinoma. Rheumatol (Oxford) (2017) 56:1706. doi: 10.1093/ rheumatology/kex224

20. Ozawa Y, Hiroshima M, Maki H, Hara M, Shibamoto Y. Imaging Findings of Lesions in the Middle and Posterior Mediastinum. Jpn J Radiol (2021) 39:1531. doi: 10.1007/s11604-020-01025-0

21. Chetty R, Serra S. SMARCA Family of Genes. J Clin Pathol (2020) 73:257-60. doi: 10.1136/jclinpath-2020-206451

22. Le Loarer F, Watson S, Pierron G, De Montpreville VT, Ballet S, Firmin N, et al. SMARCA4 Inactivation Defines a Group of Undifferentiated Thoracic Malignancies Transcriptionally Related to BAF-Deficient Sarcomas. Nat Genet (2015) 47:1200-5. doi: 10.1038/ng.3399

23. Crombé A, Alberti N, Villard N, Pilleul F, Buy X, Le Loarer F, et al. Imaging Features of SMARCA4-Deficient Thoracic Sarcomas: A Multi-Centric Study of 21 Patients. Eur Radiol (2019) 29:4730-41. doi: 10.1007/s00330-01906017-x

24. Yoshida A, Kobayashi E, Kubo T, Kodaira M, Motoi T, Motoi N, et al. Clinicopathological and Molecular Characterization of SMARCA4-Deficient Thoracic Sarcomas With Comparison to Potentially Related Entities. Mod Pathol (2017) 30:797-809. doi: 10.1038/modpathol.2017.11

25. Rekhtman N, Montecalvo J, Chang JC, Alex D, Ptashkin RN, Ai N, et al. SMARCA4-Deficient Thoracic Sarcomatoid Tumors Represent Primarily Smoking-Related Undifferentiated Carcinomas Rather Than Primary Thoracic Sarcomas. J Thorac Oncol (2020) 15:231-47. doi: 10.1016/ j.jtho.2019.10.023

26. Murakami N, Sakai T, Arai E, Muramatsu H, Ichikawa D, Asai S, et al. Targetable Driver Mutations in Multicentric Reticulohistiocytosis. Haematologica (2020) 105:e61-4. doi: 10.3324/haematol.2019.218735

27. Zhang B, Zhou H, Han J, Shi X, Zhang X. 18f-FDG PET/CT Findings in Multicentric Reticulohistiocytosis. Clin Nucl Med (2016) 41:333-5. doi: 10.1097/rlu.0000000000001071

28. Dietz M, Debarbieux S, Righetti M, Harou O, Tordo J. Paraneoplastic Multicentric Reticulohistiocytosis on 18F-FDG PET/CT Breast Carcinoma Follow-Up. Clin Nucl Med (2021) 46:e253-5. doi: 10.1097/rlu. 0000000000003417

29. Asano T, Suzutani K, Watanabe A, Honda A, Mori N, Yashiro M, et al. The Utility of FDG-PET/CT Imaging in the Evaluation of Multicentric Reticulohistiocytosis: A Case Report. Med (Baltimore) (2018) 97:e11449. doi: $10.1097 / \mathrm{md} .0000000000011449$

30. De Jonge H, Iamele L, Maggi M, Pessino G, Scotti C. Anti-Cancer AutoAntibodies: Roles, Applications and Open Issues. Cancers (Basel) (2021) 13:813. doi: 10.3390/cancers 13040813

31. Gauderon A, Roux-Lombard P, Spoerl D. Antinuclear Antibodies With a Homogeneous and Speckled Immunofluorescence Pattern are Associated With Lack of Cancer While Those With a Nucleolar Pattern With the 
Presence of Cancer. Front Med (Lausanne) (2020) 7:165 doi: 10.3389/ fmed.2020.00165

32. Wu J, Li X, Song W, Fang Y, Yu L, Liu S, et al. The Roles and Applications of Autoantibodies in Progression, Diagnosis, Treatment and Prognosis of Human Malignant Tumours. Autoimmun Rev (2017) 16:1270-81. doi: 10.1016/j.autrev.2017.10.012

33. Islam MA. Antiphospholipid Antibodies and Antiphospholipid Syndrome in Cancer: Uninvited Guests in Troubled Times. Semin Cancer Biol (2020) 64:108-13. doi: 10.1016/j.semcancer.2019.07.019

34. Zinn DJ, Eckstein O, Olsen ML, Allen CE, McClain KL. Thalidomide, a Rational Agent for Treatment of Multicentric Reticulohistiocytosis. Dermatol Case Rep (2019) 4:152. doi: 10.35248/2684-124x.19.4.152

35. Kim S, Khatchaturian EM, Dehesa L. Multicentric Reticulohistiocytosis: A Case Report With Response to Adalimumab. Clin Case Rep (2020) 8:1560-3. doi: $10.1002 / \mathrm{ccr} 3.2926$

36. Bruscas Izu C, Hörndler Argarate C, García Latasa de Araníbar FJ. Multicentric Reticulohistiocytosis: A Case Report Treated With Tofacitinib. Med Clin (Barc) (2020) 156:310-1. doi: 10.1016/j.medcli.2019.12.011

37. Niaki OZ, Penn E, Scott DA, Cobos G, Vleugels RA, Weinblatt ME. Treatment of Severe Multicentric Reticulohistiocytosis With
Upadacitinib. JAMA Dermatol (2021) 157:735-7. doi: 10.1001/jamadermatol. 2021.0996

Conflict of Interest: The authors declare that the research was conducted in the absence of any commercial or financial relationships that could be construed as a potential conflict of interest.

Publisher's Note: All claims expressed in this article are solely those of the authors and do not necessarily represent those of their affiliated organizations, or those of the publisher, the editors and the reviewers. Any product that may be evaluated in this article, or claim that may be made by its manufacturer, is not guaranteed or endorsed by the publisher.

Copyright (c) 2022 Tang, Wang, Xia, Wang, Zhao and Liu. This is an open-access article distributed under the terms of the Creative Commons Attribution License (CC BY). The use, distribution or reproduction in other forums is permitted, provided the original author(s) and the copyright owner(s) are credited and that the original publication in this journal is cited, in accordance with accepted academic practice. No use, distribution or reproduction is permitted which does not comply with these terms. 